Research Article

\title{
Yoga Training Impacts Physical Function 12 Months Post Intervention for Care Partners of those with Mild Cognitive Impairment
}

Melanie J. Chandler ${ }^{1,}{ }^{+},{ }^{*}$, Michelle Graff Radford ${ }^{1,} \dagger$, Pauline Lucas ${ }^{2}+$, Dona E.C. Locke ${ }^{2,} \dagger$, Julia Crook $^{1,}+$, Colleen Ball ${ }^{1,}$, , Maria Caselli ${ }^{2,}$, , Angela Lunde ${ }^{3,}+$, Vaishali Phatak ${ }^{4,}+$, Glenn E. Smith ${ }^{5}$ $+$

1. Mayo Clinic Florida, 4500 San Pablo Rd, Jacksonville, FL, USA; E-Mails: chandler.melanie@mayo.edu; graffradford.michelle@mayo.edu; crook.julia@mayo.edu; ball.colleen@mayo.edu

2. Mayo Clinic Arizona, 13400 E. Shea Blvd., Scottsdale, AZ, USA; E-Mails: lucas.pauline@mayo.edu; locke.dona@mayo.edu; caselli.maria@mayo.edu

3. Mayo Clinic Minnesota, 200 1st St. SW, Rochester, MN, USA; E-Mail: lunde.angela@mayo.edu

4. University of Nebraska Medical Center, 988425 Nebraska Medical Center, Omaha, Nebraska, USA; E-Mail: vaishali.phatak@unmc.edu

5. University of Florida, 1225 Center Dr. Rm 3154, Gainesville, FL, USA; E-Mail: glennsmith@phhp.ufl.edu

+ These authors contributed equally to this work.

* Correspondence: Melanie Chandler; E-Mail: chandler.melanie@mayo.edu

Academic Editor: Marieke Van Puymbroeck

Collection: Yoga in Older Adults

OBM Geriatrics

2021, volume 5 , issue 1

doi:10.21926/obm.geriatr.2101147
Received: August 30, 2020

Accepted: December 15, 2020

Published: January 04, 2021

\begin{abstract}
Physical exercise has been demonstrated to help maintain cognition in people with Mild Cognitive Impairment (pwMCl). We previously demonstrated yoga's benefit in maintenance of memory related activities of daily living in pwMCl. Our research also has shown yoga helps sustain positive psychological well-being of care partners at 12 months. In this analysis, we
\end{abstract}

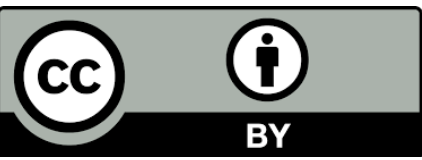

(C) 2021 by the author. This is an open access article distributed under the conditions of the Creative Commons by Attribution License, which permits unrestricted use, distribution, and reproduction in any medium or format, provided the original work is correctly cited. 
sought to examine the impact of yoga training on physical performance in pwMCl and their care partners. In this multisite, randomized clinical trial, we enrolled 272 patients meeting National Institute on Aging-Alzheimer's Association criteria for $\mathrm{MCl}$ and a care partner. The intervention program was modeled on the Mayo Clinic HABIT Healthy Action to Benefit Independence and Thinking ${ }^{\circledR}$ program. Of 5 possible interventions (memory compensation training, computerized cognitive training, yoga, support group, and wellness education), 1 of 5 interventions was randomly selected to be withheld for each intervention group. This randomization allowed for the examination of the impact of receiving yoga or no yoga on physical functioning [as measured by the Short Physical Performance Battery (SPPB)] up to 1 year post intervention, as well as the comparative effectiveness on physical functioning of the various interventions. There was a general pattern of physical decline on the SPPB over the year in $\mathrm{pwMCl}$, regardless of yoga training (yoga vs. no yoga $d=0.06, p=0.79$ ). Among care partners, those who received yoga had better physical function at 12 months compared to those who did not ( $d=0.39$ points, $p=0.041)$. While not reaching statistical significance, yoga training tended to have a greater effect on SPPB than computerized cognitive training $(\mathrm{d}=$ 0.46 points, $p=.063$ ) or wellness education $(d=0.42$ points, $p=.088)$. Participation in yoga had a positive impact on physical performance outcomes at 12 month follow-up for care partners, but did not counteract general physical decline in $\mathrm{pwMCl}$.

\section{Keywords}

Behavioral interventions; caregiver; cognitive intervention; $\mathrm{MCl}$; nonpharmacologic interventions; patient preferences

\section{Introduction}

There is a well-established literature supporting the benefits of physical exercise for cognitive health in aging [1]. Physical exercise has been shown to reduce the risk of dementia [2], and improve cognitive function in older adults with Mild Cognitive Impairment ( $\mathrm{MCl})$ or dementia [3]. Much of this research has focused on the benefit of cardiovascular activity.

There is growing evidence of the many health benefits of yoga. Yoga is a mind-body practice that combines asanas (poses) with breathing and mindfulness/meditation practices. As examples, yoga has been shown to reduce depression [4] and anxiety/stress [5] in all age groups, as well as improve quality of life and sense of well-being in older individuals [6]. Improvements in psychological outcomes such as optimism, quality of life, stress, resilience, and mood have been reported in randomized controlled trials of yoga in persons with $\mathrm{MCl}(\mathrm{pwMCl})$ [7] or multicomponent mindfulness-meditation interventions that included yoga. $[8,9]$

In terms of cognitive function in normal aging, yoga was found to improve executive function and processing speed in those over age 60 in a meta-analysis of 41 studies of mind-body exercises (including yoga, Tai Chi, or Qigong) [10]. In MCl, meta-analysis of existing data suggests mind-body exercises (including tai chi, yoga, and qigong) may benefit in overall cognition, and specifically in attention, short-term memory, executive function, and visuospatial function [11]. A further review 
suggests yoga may be particularly helpful in benefitting attention, processing speed, and verbal memory [12].

In our own previous comparative outcomes research, yoga, more than cognitive rehabilitation, computerized cognitive training, support groups, or wellness education, lead to better activities of daily living ( $A D L)$ function at 12 months [13] and 18 months for pwMCl [14]. Caregiver partners who participated in yoga also experienced better mood and less anxiety and caregiver burden at 12 months [15]. Mindfulness-based stress reduction techniques, including yoga, may be helpful with stress-related problems such as anxiety and depression in caregivers, but the number of quality of studies has been low to date [16].

In terms of physical function, multiple studies have shown that yoga practice improves physical mobility and balance in those over age 60 [17]. Improvements in physical function, including strength, muscle endurance, balance, and flexibility have been reported in small, non-comparison group trials of dementia patients in care facilities [18, 19], However, not all studies have shown physical benefit from yoga in dementia patients [20]. One study reported reduced fall risk in patients with $\mathrm{MCl}$ after a yoga intervention [21]. Otherwise, there have been no studies looking at the physical function outcomes of yoga for pwMCl or their caregivers that we know of to date. Thus, in this report, we examined the impact of participation in a 10 day yoga training program and subsequent booster sessions on physical function of pwMCl and their care partners up to 12 months post training. We hypothesized that those trained in yoga would do better on tasks of motility, balance, and speed than those who experienced other behavioral interventions, but not yoga training.

\section{Materials}

This study was part of a larger comparative effectiveness of behavioral interventions in $\mathrm{MCl}$ trial that also examined the impact of the other components of Mayo Clinic's HABIT Healthy Action to Benefit Independence \& Thinking ${ }^{\circledR}$ Program, including: compensatory cognitive rehabilitation with the Memory Support System (MSS) [22], computerized cognitive training (CCT) [23], Wellness Education, and Support Groups. Full details of the study protocol and recruitment have been reported previously [24].

Unlike previous publications from this larger project, this study did not seek the comparative effectiveness of multiple interventions on a specified outcome. In contrast to other studies where any of the interventions may have impacted an outcome, for example, quality of life [13], only yoga was anticipated to impact physical functioning. As such, in this report, we compared those who were randomized to receive yoga to those who did not.

\subsection{Participants}

Between September 2014 and August 2016, we recruited 272 dyads consisting of a pwMCl and partner through clinical services at Mayo Clinic in campuses in Rochester, Minnesota; Scottsdale, Arizona; and Jacksonville, Florida; as well as at the University of Washington in Seattle, Washington. Eligible study participants were diagnosed with amnestic $\mathrm{MCl}$, which could be either"single-domain" where the cognitive impairment was solely memory loss or "multi-domain" where memory was affected plus at least one other cognitive domain (language, visuospatial, or executive function) [25]. Inclusion criteria comprised a Clinical Dementia Rating [26] of 0.5 or lower, not taking or stable on 
nootropic medication for $>3$ months, fluent in English, and attendance with a cognitively unimpaired (Mini-Mental Status Examination [27] score >24) care partner with at least twice-weekly contact with the pwMCl. Exclusion criteria included current participation in another treatmentrelated clinical trial or major auditory, visual, or motor impairment impacting ability to participate in the program. PwMCl completed the Dementia Rating Scale-2 [28] as a measure of general cognitive function at baseline. All participants provided written informed consent.

\subsection{Intervention, Randomization, and Outcomes}

Subjects with $\mathrm{MCl}$ were randomly assigned to receive yoga or not as part of a multicomponent behavioral intervention program. They also randomly received 3 of the other possible 4 interventions (MSS, CCT, Support Groups, or Wellness Education). If not randomized to yoga, they received all 4 of the other interventions. Each of these components was originally chosen on a theoretical basis because each had support individually in the literature for effectiveness compared to no-treatment controls across a variety of outcomes (eg, cognitive functioning, QOL, mood, partner burden) [29, 30].

The intervention occurred for 10 business days in a row over 2 weeks; one hour was allotted for each intervention each day, for a 4 hour day. A 1-day booster session was provided at 6 months and 12 months post initial training delivery, and included a refresher for each of the 4 interventions originally provided (described further in our previous publication) [24] Each group session consisted of up to 20 dyads. The randomization assignment was per group session so that all members of the group were receiving the same 4 out of 5 possible interventions (Yoga, MSS, CCT, Support Groups, or Wellness Education) to help account for treatment diffusion. Group assignment was concealed from participants until they arrived for their first day of the program. Those collecting the physical outcome data were blind to group randomization.

The intervention utilized Hatha yoga. Classes included poses (asanas), breathing exercises (pranayama), meditation and relaxation exercises, and closed with affirmation or gratitude sharing. The sessions used an armless, sturdy chair placed on top of a yoga mat. We were able to adapt the yoga practice as needed so that deconditioned participants or those with imbalance could sit on chairs for some asanas (poses) and use the chair for support for balance during other standing poses and for other parts of the sequence. Our instructors had at least 200 hours of training and were Yoga Alliance certified. The appropriately sequenced yoga practice met the American College of Sports Medicine recommendation for older adults for muscle strengthening and flexibility.

Most clinical trials of yoga include group classes during the delivery phase supported by home practice in the maintenance phase. As such we provided a customized yoga DVD to participants who randomized to yoga training and encouraged its continued use and practice after the program. The DVD included sections on the following: poses, modifications, benefits, breathing, and meditation practices. Participants and partners were encouraged to strive for 150 minutes per week of physical activity (not limited to just yoga). We collected follow-up adherence questionnaires about time spent in physical activity at 6 and 12 months post intervention. Participants and partners whom engaged in at least 150 minutes of physical activity per week were assigned to the post-intervention 'adherent' category, <60 min per week were assigned to the 'non-adherent' category, and those who fell between the required standards for adherent and non-adherent were assigned to the 'indeterminate' category [31]. 
All participants (pwMCl and the care partners) completed the Short Physical Performance Battery [32] (SPPB) at baseline, treatment end, and 12 months post-intervention. The SPPB includes a timed 400-meter walk (speed), standing with feet positioned side by side, semi-tandem and full tandem stance (balance), and a timed arms-folded rise from seated-to-standing $\times 4$ (strength). Each section (speed, balance, strength) was worth 4 possible points for a maximum total score of 12 . Higher scores indicate better physical performance. All outcome data was collected prior to the commencement of the 12 month booster session.

\subsection{Statistical Analysis}

Longitudinal mixed-effects regression models, separately for pwMCl and care partners, were used to evaluate the impact of yoga on SPPB scores at 12 months using data baseline, end of treatment, and 12-month follow-up. Baseline SPPB score was modelled with fixed effects for age, sex, and site. The mean change in SPPB score was modelled with fixed effects for study arm (no CCT, no MSS, no support groups, no wellness, and no yoga), age, and sex. Person-specific random effects were included to account for multiple measurements over time. For our primary aim of evaluating the impact of yoga versus no yoga on SPPB score at 12 months, we focused on estimation of the difference $(d)$ between the average of the means in the four arms that included yoga $(\mu 1+\mu 2+\mu 3$ $+\mu 4) / 4$ and the mean in the no yoga arm $(\mu 5)$ by expressing it as a linear contrast: $d=0.25 \mu 1+$ $0.25 \mu 2+0.25 \mu 3+0.25 \mu 4-1 \mu 5$. We constructed $95 \%$ confidence intervals $(\mathrm{Cl})$ using the profile likelihood method and corresponding likelihood ratio tests. We adjusted for multiple testing with Westfall stepwise adjustment. Effect sizes (ES) were expressed as multiples of the baseline standard deviation of the SPPB score. Analyses were performed using R statistical software, version 3.2.3 ( $R$ Foundation for Statistical Computing).

The conduct of the study was reviewed and approved by the Institutional Review Boards at Mayo Clinic (14-000885) and the University of Washington (49235). CONSORT reporting guidelines were followed. Trial is registered at ClinicalTrials.gov, identifier: NCT02265757.

\section{Results}

We enrolled 272 participants in this study. Recruitment information has been described in detail elsewhere [13]. All but 44 participants completed the study through 12 month follow up (83.8\% retention). There was no significant difference in attrition between those randomized to yoga ( $82.4 \%$ retention) or no-yoga (89.3\% retention). There were no meaningful differences in demographics or general cognitive status across sites or groups at baseline (Table 1). No adverse effects were detected as a result of this study.

Table 1 Demographic overview for pwMCl and their care partners.

\begin{tabular}{llllll}
\hline No Yoga & No CCT & $\begin{array}{l}\text { No Wellness } \\
\text { Education }\end{array}$ & $\begin{array}{l}\text { No Support } \\
\text { Groups }\end{array}$ & No MSS \\
\hline pwMCl & $(n=56)$ & $(n=54)$ & $(n=52)$ & $(n=53) \quad(n=57)$ \\
\hline
\end{tabular}




\begin{tabular}{|c|c|c|c|c|c|}
\hline Age, Mean (SD) & $74.3(7.3)$ & $75.8(8.0)$ & $76.7(7.3)$ & $75.1(7.3)$ & 74.1 (7.9) \\
\hline Education, Median (range) & $17(9-20)$ & $16(12-20)^{1}$ & $16(12-20)$ & $16(10-20)$ & $16(6-20)$ \\
\hline Male, No. \% & $34(61 \%)$ & $33(61 \%)$ & $30(58 \%)$ & $32(60 \%)$ & $31(54 \%)$ \\
\hline Race non-white, No. (\%)* & $3(5 \%)$ & $1(2 \%)$ & $1(2 \%)$ & $2(4 \%)$ & $5(9 \%)$ \\
\hline DRS-2 total, Mean (SD) & $130.1(8.7)^{7}$ & $127.5(9.3)$ & $128.0(8.0)^{1}$ & $130.9(7.6)$ & $129.9(8.8)^{2}$ \\
\hline Care Partner & $(n=56)$ & $(n=54)$ & $(n=52)$ & $(n=53)$ & $(n=57)$ \\
\hline Age, mean (SD) & $70.9(9.5)$ & $70.9(9.5)$ & $70.3(10.4)$ & $71.6(8.8)$ & $67.6(12.2)$ \\
\hline Male, No. (\%) & $18(32 \%)$ & $15(28 \%)$ & $18(35 \%)$ & $18(34 \%)$ & $16(29 \%)^{1}$ \\
\hline $\begin{array}{l}\text { Partner relationship, No. } \\
\text { (\%) }\end{array}$ & & & & & \\
\hline Spouse or partner & $52(93 \%)$ & $44(81 \%)$ & $42 / 50(84 \%)$ & $48(91 \%)$ & $43 / 55(78 \%)$ \\
\hline Son or daughter & $2(4 \%)$ & $6(11 \%)$ & $4 / 50(8 \%)$ & $3(6 \%)$ & $8 / 55(15 \%)$ \\
\hline
\end{tabular}

Note: Number in superscript reflects missing case numbers in that data cell compared to the overall group sample. Abbreviations: $\mathrm{CCT}=$ computerized cognitive training; $\mathrm{MSS}=$ Memory Support System; $\mathrm{pwMCl}=$ persons with $\mathrm{MCl} ; \mathrm{N}=$ number of persons with available data; $\mathrm{SD}=$ standard deviation. Higher scores translate to better physical performance.

Table 2 shows a summary of the baseline patient and care partner SPPB total score according to study arm. There were no meaningful differences in baseline SPPB total scores between study arms for pwMCl (likelihood ratio $p=0.46$ ) or care partners (likelihood ratio $p=.63$ ). A summary of the raw changes in SPPB scores from baseline to 12 months is also included in Table 2.

Table 2 Short Physical Performance Battery total scores at baseline and change at 12 months.

\begin{tabular}{|c|c|c|c|c|c|}
\hline & No Yoga & No CCT & $\begin{array}{l}\text { No Wellness } \\
\text { Education }\end{array}$ & $\begin{array}{l}\text { No Support } \\
\text { Groups }\end{array}$ & No MSS \\
\hline $\begin{array}{l}\text { No. of dyads } \\
\text { pwMCI }\end{array}$ & 56 & 54 & 52 & 53 & 57 \\
\hline Baseline & $N=55$ & $N=51$ & $N=47$ & $N=52$ & $N=50$ \\
\hline Mean (SD) & $10.38(1.74)$ & $10.20(1.94)$ & $9.87(1.68)$ & $10.13(1.78)$ & $10.60(1.56)$ \\
\hline Change at EOT & $N=52$ & $N=47$ & $N=34$ & $N=50$ & $N=46$ \\
\hline Mean (SD) & $0.21(1.11)$ & $-0.30(1.30)$ & $0.12(1.55)$ & $0.36(1.14)$ & $-0.24(0.97)$ \\
\hline Change at $12 \mathrm{mo}$ & $N=39$ & $N=33$ & $N=34$ & $N=36$ & $N=35$ \\
\hline
\end{tabular}




\begin{tabular}{llllll} 
Mean (SD) & $-0.74(1.76)$ & $-0.91(1.70)$ & $-0.71(1.80)$ & $-0.06(1.55)$ & $-0.86(1.31)$ \\
Care Partner & & & & & \\
Baseline & $\mathrm{N}=53$ & $\mathrm{~N}=52$ & $\mathrm{~N}=46$ & $\mathrm{~N}=53$ & $\mathrm{~N}=51$ \\
Mean (SD) & $10.85(1.38)$ & $11.00(1.39)$ & $10.76(1.25)$ & $11.04(1.22)$ & $11.14(1.39)$ \\
Change at EOT & $\mathrm{N}=48$ & $\mathrm{~N}=49$ & $\mathrm{~N}=31$ & $\mathrm{~N}=52$ & $\mathrm{~N}=47$ \\
Mean (SD) & $0.06(1.16)$ & $0.08(0.95)$ & $0.03(1.66)$ & $0.40(0.91)$ & $-0.02(1.13)$ \\
Change at 12 mo & $\mathrm{N}=38$ & $\mathrm{~N}=33$ & $\mathrm{~N}=31$ & $\mathrm{~N}=34$ & $\mathrm{~N}=37$ \\
Mean (SD) & $-0.39(1.53)$ & $-0.09(1.01)$ & $0.10(1.42)$ & $-0.21(0.84)$ & $-0.16(1.34)$ \\
\hline
\end{tabular}

Abbreviations: $\mathrm{CCT}=$ computerized cognitive training; $\mathrm{MSS}=$ Memory Support System; $\mathrm{pwMCl}=$ persons with $\mathrm{MCl} ; \mathrm{N}=$ number of persons with available data; $\mathrm{SD}=$ standard deviation; $\mathrm{EOT}=$ end of treatment. Higher scores translate to better physical performance. The number of pwMCI or care partners for whom the data was available did not always equal the total number of dyads in an intervention due to missing data.

In comparing SPPB total score between those randomized to the no yoga arm and those randomized to one of the other four arms that included yoga (Figure 1), there were no significant differences at end of treatment for yoga versus no yoga in pwMCl (Effect Size $-0.17,95 \% \mathrm{Cl}-0.40$ to 0.07, $\mathrm{P}=0.16$ ) or care partners (Effect Size $-0.04,95 \% \mathrm{Cl}-0.29$ to $0.21, \mathrm{P}=0.76$ ). By 12 -months post intervention, yoga did not impact SPPB total score in $\mathrm{MCl}$ patients (Effect Size $0.04,95 \% \mathrm{Cl}-0.23$ to $0.30, \mathrm{P}=.79$ ), but yoga training maintained SPPB total scores in their care partners at 12 months compared to a decline in the no yoga group (Effect Size $0.29,95 \% \mathrm{Cl} 0.01$ to $0.57, \mathrm{P}=0.04$ ). 


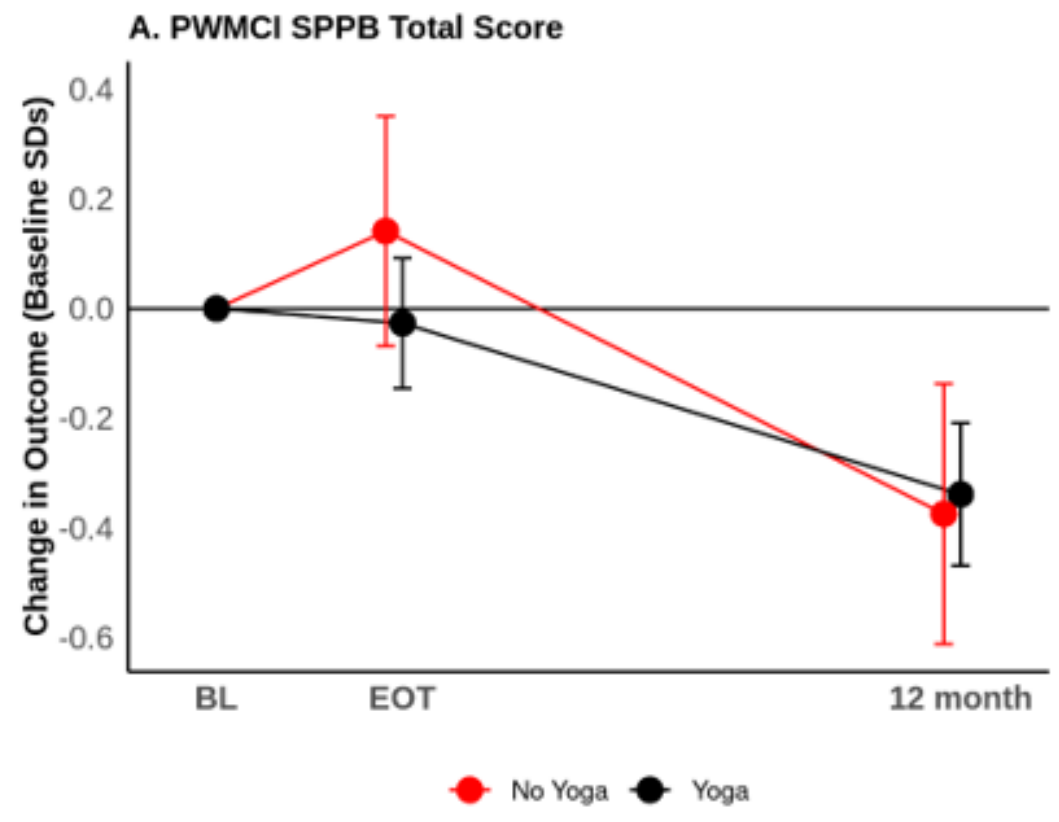

B. Care Partner SPPB Total Score

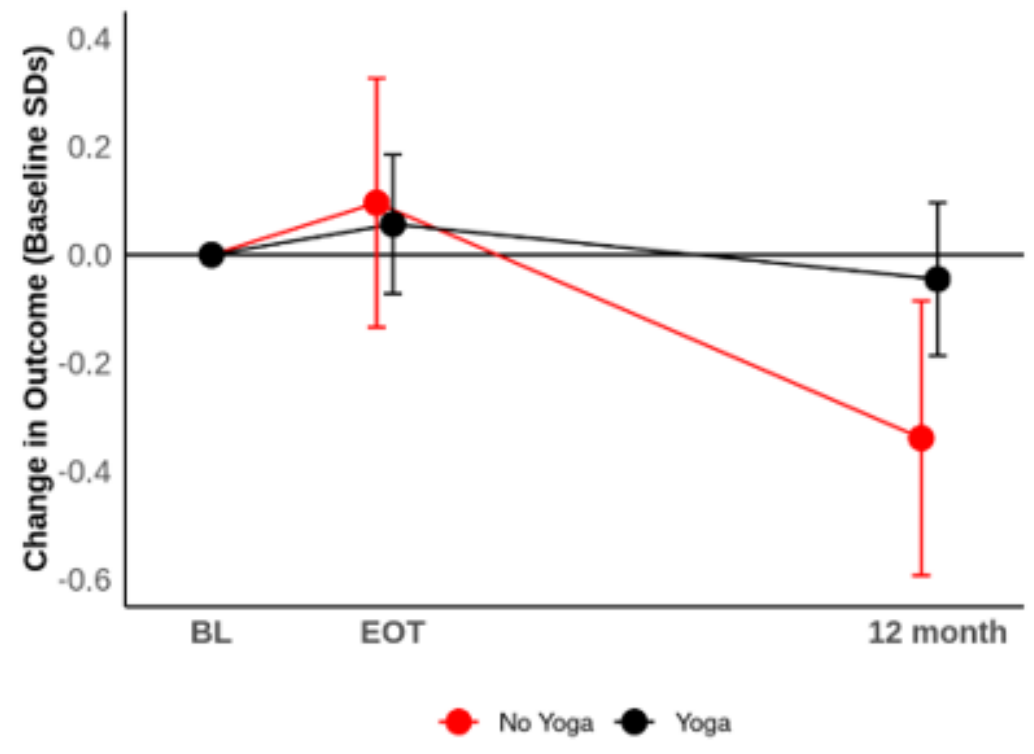

Figure 1 Effect sizes for change in Short Physical Performance Battery score for yoga vs. no yoga groups. Abbreviations: $\mathrm{BL}=$ Baseline; $\mathrm{EOT}=$ End of Treatment. Effect sizes were estimated from longitudinal mixed-effects regression models, in which a 1-unit increase in the effect size corresponded to a 1-SD improvement in outcome. Baseline SDs were 1.749 for pWMCI SPPB total score and 1.325 for care partner SPPB total score. Error bars represent $95 \%$ confidence intervals for the effect sizes.

Comparisons of the SPPB total score between the 5 study arms are shown in Figure 2 as well as Tables 3 (patients) and 4 (care partners). We found that CCT and wellness education had more of a positive effect on SPPB total score compared to support groups for our $\mathrm{MCl}$ patients, however these associations were not statistically significant after adjustment for multiple testing (Table 3). Among the care partners enrolled in our study, there were not any statistically significant differences in 
SPPB total score between the five components, however it is worth noting that yoga had the largest effect size in comparison to all other interventions (Table 4).

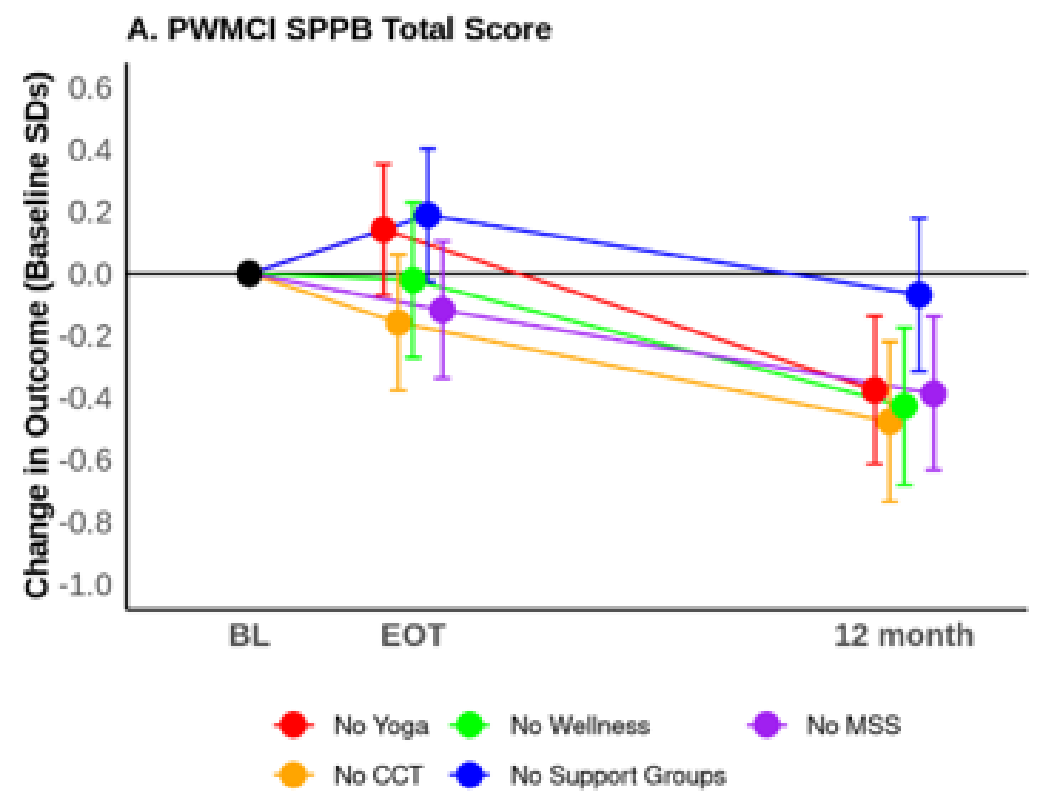

B. Care Partner SPPB Total Score

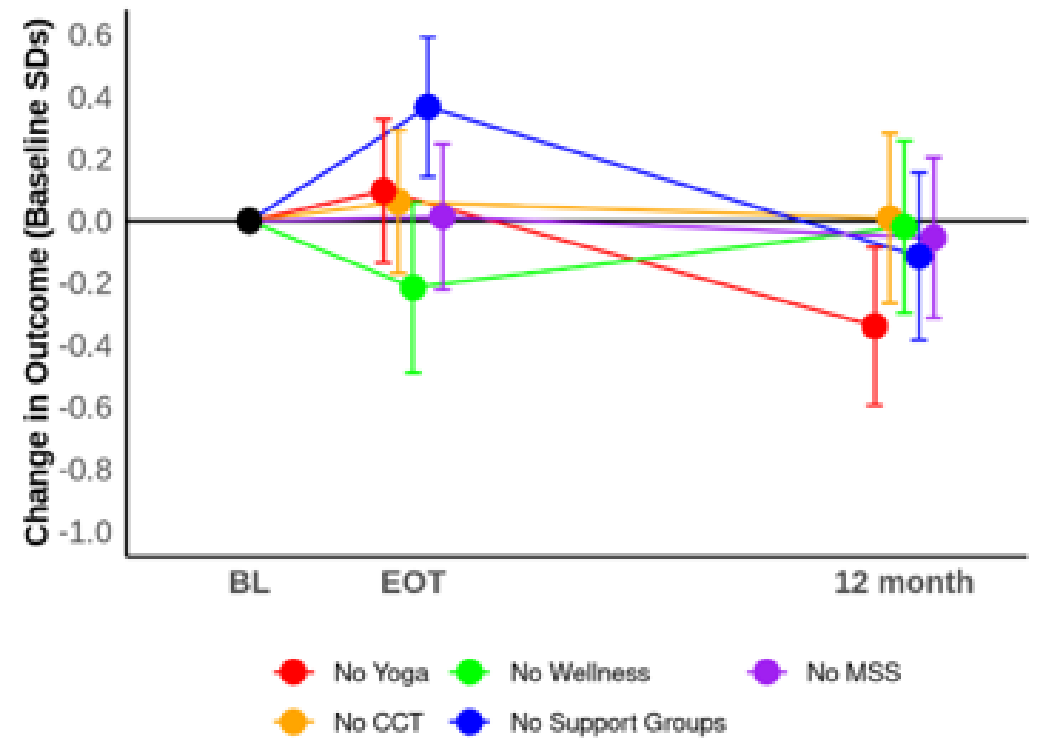

Figure 2 Comparative effectiveness of interventions on Short Physical Performance Battery score. Abbreviations: $\mathrm{BL}=$ Baseline; $\mathrm{CCT}=$ computerized cognitive training; $\mathrm{EOT}$ = End of Treatment; MSS= memory support system. Error bars represent 95\% confidence intervals for the effect sizes. 
Table 3 Comparative Incremental Effect on Patient SPPB Total Score at 12 Months for Pairs of HABIT Components.

\begin{tabular}{llllll}
\hline Component & $\begin{array}{l}\text { Compared } \\
\text { to }\end{array}$ & $\begin{array}{l}\text { Difference in SPPB at } \\
12 \text { mo } \\
(95 \% \mathrm{Cl}), \text { points }\end{array}$ & $\begin{array}{l}\text { Effect Size } \\
(95 \% \mathrm{Cl})\end{array}$ & $\begin{array}{l}\text { Original } \\
P \text { value }\end{array}$ & $\begin{array}{l}\text { Adjusted } \\
P \text { value }\end{array}$ \\
\hline CCT & Support & $0.71(0.10$ to 1.33$)$ & $0.41(0.06$ to 0.76$)$ & 0.022 & 0.15 \\
Wellness & Support & $0.63(0.02$ to 1.23$)$ & $0.36(0.01$ to 0.71$)$ & 0.043 & 0.24 \\
MSS & Support & $0.56(-0.04$ to 1.16$)$ & $0.32(-0.03$ to 0.66$)$ & 0.070 & 0.33 \\
Yoga & Support & $0.54(-0.05$ to 1.12$)$ & $0.31(-0.03$ to 0.64$)$ & 0.074 & 0.33 \\
CCT & Yoga & $0.18(-0.42$ to 0.78$)$ & $0.10(-0.24$ to 0.45$)$ & 0.56 & 0.94 \\
CCT & MSS & $0.16(-0.46$ to 0.78$)$ & $0.09(-0.26$ to 0.44$)$ & 0.61 & 0.95 \\
Wellness & Yoga & $0.09(-0.51$ to 0.69$)$ & $0.05(-0.29$ to 0.39$)$ & 0.77 & 0.99 \\
CCT & Wellness & $0.09(-0.53$ to 0.71$)$ & $0.05(-0.31$ to 0.41$)$ & 0.78 & 0.99 \\
Wellness & MSS & $0.07(-0.54$ to 0.68$)$ & $0.04(-0.31$ to 0.39$)$ & 0.82 & 0.99 \\
MSS & Yoga & $0.02(-0.57$ to 0.61$)$ & $0.01(-0.33$ to 0.35$)$ & 0.95 & 0.99 \\
\hline
\end{tabular}

Abbreviations: $\mathrm{Cl}=$ confidence interval $\mathrm{CCT}=$ Computerized Cognitive Training, $\mathrm{MSS}=$ Memory Support System. To ease interpretation, the two components compared are ordered such that the first component listed has a better estimated mean outcome at 12 months than the second component. Effect size is the difference in mean SPPB score at 12 months expressed as multiples of the baseline standard deviation of the SPPB score. Both original and multiple-test adjusted $P$ values are shown.

Table 4 Comparative Incremental Effect on Informant SPPB Total Score at 12 Months for Pairs of HABIT Components.

\begin{tabular}{lllllll}
\hline Component & Compared to & $\begin{array}{l}\text { Difference in SPPB at } \\
12 \text { mo }(95 \% \quad \mathrm{Cl}) \\
\text { points }\end{array}$ & $\begin{array}{l}\text { Effect Size } \\
(95 \% \mathrm{Cl})\end{array}$ & $\begin{array}{l}\text { Original } \\
P \text { value }\end{array}$ & $\begin{array}{l}\text { Adjusted } \\
P \text { value }\end{array}$ \\
\hline Yoga & CCT & $0.46(-0.02$ to 0.94$)$ & $0.35(-0.02$ to 0.71$)$ & 0.063 & 0.34 \\
Yoga & Wellness & $0.42(-0.06$ to 0.91$)$ & $0.32(-0.05$ to 0.69$)$ & 0.088 & 0.41 \\
Yoga & MSS & $0.38(-0.09$ to 0.85$)$ & $0.28(-0.07$ to 0.64$)$ & 0.12 & 0.48 \\
Yoga & Support & $0.30(-0.18$ to 0.78$)$ & $0.22(-0.14$ to 0.59$)$ & 0.23 & 0.68 \\
Support & CCT & $0.16(-0.33$ to 0.66$)$ & $0.12(-0.25$ to 0.50$)$ & 0.52 & 0.92 \\
Support & Wellness & $0.13(-0.37$ to 0.63$)$ & $0.10(-0.28$ to 0.47$)$ & 0.62 & 0.95 \\
MSS & CCT & $0.08(-0.40$ to 0.57$)$ & $0.06(-0.30$ to 0.43$)$ & 0.74 & 0.98
\end{tabular}




\begin{tabular}{llllll} 
Support & MSS & $0.08(-0.40$ to 0.56$)$ & $0.06(-0.31$ to 0.43$)$ & 0.75 & 0.98 \\
MSS & Wellness & $0.05(-0.44$ to 0.54$)$ & $0.04(-0.33$ to 0.41$)$ & 0.85 & 0.98 \\
Wellness & CCT & $0.04(-0.47$ to 0.54$)$ & $0.03(-0.35$ to 0.41$)$ & 0.89 & 0.98 \\
\hline
\end{tabular}

Abbreviations: $\mathrm{Cl}=$ confidence interval, $\mathrm{CCT}=$ Computerized Cognitive Training, $\mathrm{MSS}=$ Memory Support System. To ease interpretation, the two components compared are ordered such that the first component listed has a better estimated mean outcome at 12 months than the second component. Effect size is the difference in mean SPPB score at 12 months expressed as multiples of the baseline standard deviation of the SPPB score. Both original and multiple-test adjusted $P$ values are shown.

\section{Discussion}

$\mathrm{MCl}$ is a syndrome that impacts not just those diagnosed with the disorder, but also their loved ones. Here, we examined the impact of Hatha yoga training on the physical functioning of both pwMCl and their care partners out to 12 months after the intervention. Our previous work suggested that yoga training was the most effective of five potential interventions offered (including compensatory calendar training, computerized cognitive training, wellness education, support groups, and yoga) in maintenance of memory related activities of daily living out to 12 and 18 months $[13,14]$. In this study, there was no significant improvement in physical functioning for pwMCl 12 months after yoga training (or any of the other interventions). Rather, pwMCl experienced a gradual decline in physical functioning over the 12 month follow-up, regardless of intervention group. This is consistent with prior reporting that a physical decline often co-occurs with the cognitive decline experienced in many with $\mathrm{MCl}[33,34]$. Hatha yoga training does not appear to overcome this natural decline.

Yoga training did maintain care partner physical functioning up to the 12 month follow up period of this study when compared to other groups not receiving yoga who experienced physical decline. This is the first report of the positive impact of yoga training on physical function performance in care partners of which we are aware. There have been prior reports of emotional well-being benefits to caregivers from yoga training [35]. In this same study cohort, we previously reported that yoga benefited mood, lowered anxiety, and lowered caregiver burden in care partners up to 12 month [15]. Unique to the current study, yoga training appears to not just benefit the psychological wellbeing of care partners, but also their physical well-being.

\subsection{Limitations}

Our Hatha yoga sessions were comprised of a combination of poses (asanas), breathing exercises (pranayama), meditation/relaxation exercises, and gratitude practice. We cannot determine from this current study which aspects of the Hatha yoga training were particularly beneficial to our care partners. However, it is reasonable to speculate that the asana practices that encouraged balance, flexibility, and core strength likely contributed to the physical function benefit.

While we encouraged yoga maintenance post training, we also emphasized engagement in physical activity in general. Our adherence measures tracked overall physical activity and not specifically adherence to yoga. We did not collect SPPB data at the 6 month follow-up session, and thus cannot speak to the impact of yoga training on physical function at that time point. At 12 
month follow-up, $40.0 \%$ of pwMCl and $45.9 \%$ for partners were fully "adherent" to physical activity (> 150 minutes a week), and $82.4 \%$ of $\mathrm{pwMCl}$ and $87.7 \%$ of partners were exercising at least 60 minutes a week. However, individuals may have engaged in yoga, a different physical activity, or a combination of the two in the post intervention maintenance period. This limits our ability to speak to how much maintenance of yoga alone after the initial training period impacted physical function. Paper and pencil adherence questionnaires further are subject to reporting bias of recent physical activity. This potential bias is compounded in our study of pwMCl with memory impairments, and, at times, care partners reported on both the pwMCl and themselves. It is important to note that participants and care partners were only trained in yoga during the intervention regardless of the weakness of our adherence measure to capture their yoga-specific adherence. Further, yoga may have been more physically beneficial to pwMCl if more support of its continued practice was provided in the maintenance year following training delivery.

\section{Conclusions}

Yoga does not appear to improve physical function of patients with $\mathrm{MCl}$, but yoga may maintain physical function of their care partners.

\section{Author Contributions}

Drs. Chandler, Smith, and Locke were involved in the development of the research protocol, served as Mayo Clinic site Pls, and led patient recruitment during this study. Dr. Phatak served as site $\mathrm{PI}$ and recruiter at the University of Washington. Dr. Crook and Ms. Ball served as statistical support and led writing of the statistical methods, results, and tables/figures. Dr. Lucas, Ms. GraffRadford, Ms. Lunde, and Ms. Caselli were yoga instructors who helped inform the yoga programming and implementation. While Dr. Chandler took the lead role in writing the manuscript, all authors contributed to its final editing and approved the final draft.

\section{Funding}

Research reported in this manuscript was primarily funded through a Patient-Centered Outcomes Research Institute (PCORI) Award (CER-1306-01897). The statements in this publication are solely the responsibility of the authors and do not represent the views of PCORI, its Board of Governors, or the Methodology Committee. The funding source provided feedback on the study design and oversight to the protocol implementation.

\section{Competing Interests}

The authors have declared that no competing interests exist.

\section{References}

1. Northey JM, Cherbuin N, Pumpa KL, Smee DJ, Rattray B. Exercise interventions for cognitive function in adults older than 50: A systematic review with meta-analysis. Br J Sports Med. 2018; 52: 154-160. 
2. Lee J. The relationship between physical activity and dementia: A systematic review and metaanalysis of prospective cohort studies. J Gerontol Nurs. 2018; 44: 22-29.

3. Law CK, Lam FM, Chung RC, Pang MY. Physical exercise attenuates cognitive decline and reduces behavioural problems in people with mild cognitive impairment and dementia: $A$ systematic review. J Physiother. 2020; 66: 9-18.

4. Mathersul DC, Rosenbaum S. The roles of exercise and yoga in ameliorating depression as a risk factor for cognitive decline. Evid Based Complement Alternat Med. 2016; 2016: 4612953.

5. Pascoe MC, Bauer IE. A systematic review of randomised control trials on the effects of yoga on stress measures and mood. J Psychiatr Res. 2015; 68: 270-282.

6. Tulloch A, Bombell H, Dean C, Tiedemann A. Yoga-based exercise improves health-related quality of life and mental well-being in older people: A systematic review of randomised controlled trials. Age Ageing. 2018; 47: 537-544.

7. Eyre HA, Siddarth P, Acevedo B, Van Dyk K., Paholpak P, Ercoli L, et al. A randomized controlled trial of Kundalini yoga in mild cognitive impairment. Int Psychogeriatr. 2017; 29: 557-567.

8. Wells RE, Kerr CE, Wolkin J, Dossett M, Davis RB, Walsh J, et al. Meditation for adults with mild cognitive impairment: A pilot randomized trial. J Am Geriatr Soc. 2013; 61: 642-645.

9. Wetherell JL, Hershey T, Hickman S, Tate SR, Dixon D, Bower ES, et al. Mindfulness-based stress reduction for older adults with stress disorders and neurocognitive difficulties: A randomized controlled trial. J Clin Psychiatry. 2017; 78: 734-743.

10. Chan JS, Deng K, Wu J, Yan JH. Effects of meditation and mind-body exercises on older adults' cognitive performance: A meta-analysis. Gerontologist. 2019; 59: e782-e790.

11. Zou L, Loprinzi PD, Yeung AS, Zeng N, Huang T. The beneficial effects of mind-body exercises for people with mild cognitive impairment: A systematic review with meta-analysis. Arch Phys Med Rehabil. 2019; 100: 1556-1573.

12. Brenes GA, Sohl S, Wells RE, Befus D, Campos CL, Danhauer SC. The effects of yoga on patients with mild cognitive impairment and dementia: A scoping review. Am J Geriatr Psychiatry. 2019; 27: 188-197.

13. Chandler MJ, Locke DE, Crook JE, Fields JA, Ball CT, Phatak VS, et al. Comparative effectiveness of behavioral interventions on quality of life for older adults with mild cognitive impairment: $A$ randomized clinical trial. JAMA Netw Open. 2019; 2: e193016.

14. Shandera-Ochsner A, Chandler MJ, Locke DE, et al. Comparative effects of physical exercise and other behavioral interventions on functional status outcomes in mild cognitive impairment. J Int Neuropsychol Soc. in press.

15. Amofa P, Locke DE, Chandler M, Crook JE, Ball CT, Phatak V, et al. Comparative effectiveness of behavioral interventions to prevent or delay dementia: One-year partner outcomes. J Prev Alzheimer's Dis. 2020; 8: 33-40.

16. Liu Z, Sun YY, Zhong BL. Mindfulness-based stress reduction for family carers of people with dementia. Cochrane Database Syst Rev. 2018; 8: cd012791.

17. Youkhana S, Dean CM, Wolff M, Sherrington C, Tiedemann A. Yoga-based exercise improves balance and mobility in people aged 60 and over: A systematic review and meta-analysis. Age Ageing. 2016; 45: 21-29.

18. Fan JT, Chen KM. Using silver yoga exercises to promote physical and mental health of elders with dementia in long-term care facilities. Int Psychogeriatr. 2011; 23: 1222-1230. 
19. McCaffrey R, Park J, Newman D, Hagen D. The effect of chair yoga in older adults with moderate and severe Alzheimer's disease. Res Gerontol Nurs. 2014; 7: 171-177.

20. Litchke LG, Hodges JS, Reardon RF. Benefits of chair yoga for persons with mild to severe Alzheimer's disease. Act Adapt Aging. 2012; 36: 317-328.

21. Farhang M, Miranda-Castillo C, Rubio M, Furtado G. Impact of mind-body interventions in older adults with mild cognitive impairment: A systematic review. Int Psychogeriatr. 2019; 31: 643666.

22. Greenaway MC, Duncan NL, Smith GE. The memory support system for mild cognitive impairment: Randomized trial of a cognitive rehabilitation intervention. Int J Geriatr Psychiatry. 2013; 28: 402-409.

23. Barnes DE, Yaffe K, Belfor N, Jagust WJ, DeCarli C, Reed BR, et al. Computer-based cognitive training for mild cognitive impairment: Results from a pilot randomized, controlled trial. Alzheimer Dis Assoc Disord. 2009; 23: 205-210.

24. Smith G, Chandler M, Locke DE, Fields J, Phatak V, Crook J, et al. Behavioral interventions to prevent or delay dementia: Protocol for a randomized comparative effectiveness study. JMIR Res Protoc. 2017; 6: e223.

25. Albert MS, DeKosky ST, Dickson D, Dubois B, Feldman HH, Fox NC, et al. The diagnosis of mild cognitive impairment due to Alzheimer's disease: Recommendations from the National Institute on Aging-Alzheimer's Association workgroups on diagnostic guidelines for Alzheimer's disease. Alzheimers Dement. 2011; 7: 270-279.

26. Morris JC. Clinical dementia rating: A reliable and valid diagnostic and staging measure for dementia of the Alzheimer type. Int Psychogeriatr. 1997; 9: 173-176.

27. Folstein MF, Folstein SE, McHugh PR. "Mini-mental state": A practical method for grading the cognitive state of patients for the clinician. J Psychiatr Res. 1975; 12: 189-198.

28. Jurica PJ, Leitten CL, Mattis S. Dementia Rating Scale-2: Professional manual. North Florida: Psychological Assessment Resources; 2001.

29. Chandler MJ, Parks AC, Marsiske M, Rotblatt LJ, Smith GE. Everyday impact of cognitive interventions in mild cognitive impairment: A systematic review and meta-analysis. Neuropsychol Rev. 2016; 26: 225-251.

30. Rodakowski J, Saghafi E, Butters MA, Skidmore ER. Non-pharmacological interventions for adults with mild cognitive impairment and early stage dementia: An updated scoping review. Mol Aspects Med. 2015; 43: 38-53.

31. Sr Amofa PA, DeFeis B, De Wit L, O'Shea D, Mejia A, Chandler M, et al. Functional ability is associated with higher adherence to behavioral interventions in mild cognitive impairment. Clin Neuropsychol. 2020; 34: 937-955.

32. Guralnik JM, Simonsick EM, Ferrucci L, Glynn RJ, Berkman LF, Blazer DG, et al. A short physical performance battery assessing lower extremity function: Association with self-reported disability and prediction of mortality and nursing home admission. J Gerontol. 1994; 49: M85M94.

33. Borges MK, de Castro Cezar NO, Siqueira AS, Yassuda M, Cesari M, Aprahamian I. The relationship between physical frailty and mild cognitive impairment in the elderly: A systematic review. J Frailty Aging. 2019; 1-6. 
34. Taylor ME, Boripuntakul S, Toson B, Close JC, Lord SR, Kochan NA, et al. The role of cognitive function and physical activity in physical decline in older adults across the cognitive spectrum. Aging Ment Health. 2019; 23: 863-871.

35. Danucalov MA, Kozasa EH, Ribas KT, Galduróz JC, Garcia MC, Verreschi IT, et al. A yoga and compassion meditation program reduces stress in familial caregivers of Alzheimer's disease patients. Evid Based Complement Alternat Med. 2013; 2013: 513149.

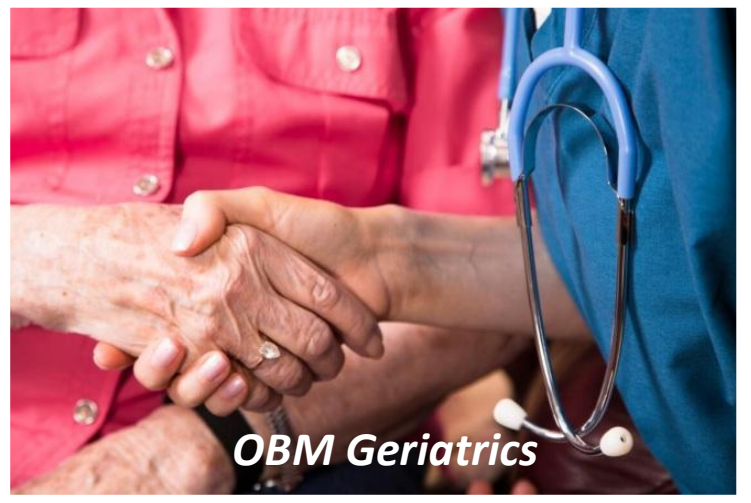

Enjoy $O B M$ Geriatrics by:

1. Submitting a manuscript

2. Joining in volunteer reviewer bank

3. Joining Editorial Board

4. Guest editing a special issue

For more details, please visit: http://www.lidsen.com/journals/geriatrics 